\title{
Hepatitis B virus core protein dimer-dimer interface is critical for viral replication
}

\author{
CHANG-LONG ZHENG ${ }^{1}$, YONG-MEI FU ${ }^{1}$, ZHAN-XUE XU ${ }^{2}$, YONG ZOU ${ }^{3}$ and KAI DENG ${ }^{4}$ \\ ${ }^{1}$ Department of Emergency, The Third Affiliated Hospital of Sun Yat-Sen University, Guangzhou, Guangdong 510630; \\ ${ }^{2}$ Institute of Human Virology and Key Laboratory of Tropical Disease Control of Ministry of Education, \\ Zhongshan School of Medicine, Sun Yat-Sen University, Guangzhou, Guangdong 510080; ${ }^{3}$ Department of Blood Transfusion, \\ The Third Affiliated Hospital of Sun Yat-Sen University, Guangzhou, Guangdong 510630; ${ }^{4}$ Institute of Infectious Diseases, \\ Guangzhou Eighth People's Hospital, Guangzhou Medical University, Guangzhou, Guangdong 510060, P.R. China
}

Received January 31, 2018; Accepted September 7, 2018

DOI: $10.3892 / \mathrm{mmr} .2018 .9620$

\begin{abstract}
Hepatitis B virus (HBV) core protein (HBc) serves pivotal roles in the viral life cycle, particularly serving as the basic unit for capsid assembly, and is closely associated with HBV genome replication and progeny virion production. Previous studies have demonstrated that $\mathrm{HBc}$ has at least two functional interfaces; two $\mathrm{HBc}$ monomers form a homodimer via an intradimer interface, and then 90 or 120 homodimers form an icosahedral capsid via a dimer-dimer interface. In the present study, the role of the HBc dimer-dimer interface in HBV replication was investigated. A panel of residues located at the dimer-dimer interface were identified based on the crystal structure of HBc. Native gel electrophoresis and western blotting revealed that, despite mutations in the dimer-dimer interface, $\mathrm{HBc}$ formed a capsid-like structure, whereas mutations at amino acid residues 23-39 completely disrupted capsid assembly. Using denaturing gel electrophoresis, Southern and Northern blotting, and quantitative polymerase chain reaction, it was demonstrated that none of the mutations in the dimer-dimer interface supported pregenomic RNA encapsidation or DNA replication. In addition, these mutants interacted with the wild-type (WT) HBc monomer and inhibited WT genome replication and virion production in a dose-dependent
\end{abstract}

Correspondence to: Dr Yong Zou, Department of Blood Transfusion, The Third Hospital of Sun Yat-Sen University, 600 Tianhe Road, Tianhe, Guangzhou, Guangdong 510630, P.R. China

E-mail: zouyong0205@163.com

Dr Kai Deng, Institute of Infectious Diseases, Guangzhou Eighth People's Hospital, Guangzhou Medical University, 627 West Dongfeng Road, Yuexiu, Guangzhou, Guangdong 510060, P.R. China E-mail: d529532013@aliyun.com

Abbreviations: $\mathrm{HBV}$, hepatitis B virus; $\mathrm{HBc}$, hepatitis B virus core protein; pgRNA, pre-genomic RNA; cccDNA, covalently closed circular DNA; aa, amino acid; WT, wild-type

Key words: hepatitis B virus, core protein, dimer-dimer interface, capsid, replication, virion manner. However, the quantity of covalently closed circular DNA in the nucleus was not affected. The present study highlighted the importance of the HBc dimer-dimer interface for normal capsid function and demonstrated that the $\mathrm{HBc}$ dimer-dimer interface may be a novel antiviral target.

\section{Introduction}

Hepatitis B virus (HBV) infects $\sim 350$ million people worldwide and is one of the most serious public health problems (1). The virus belongs to the Hepadnaviridae family and contains a 3.2-kb partially double-stranded circular genome; HBV may be one of the smallest of microbial pathogens affecting humans (2). HBV replication is highly dependent on the accurate assembly of the capsid, which is also associated with the covalently closed circular DNA (cccDNA) reservoir for persistent infection $(3,4)$. Following translation from full-length pregenomic RNA (pgRNA), hepatitis B virus core (HBc) protein interacts with pgRNA, reverse transcriptase, and host factors to form icosahedral-shaped capsids and initiate viral replication (5).

The HBV capsid is closely associated with genome replication (3). A few small molecules, including GLS4, Huntingtin-associated protein 1 and AT130, disrupt capsid formation and inhibit viral replication (6-9). These molecules alter the structure and disrupt the function of capsids (7). In addition, capsids have been considered to mediate the regulation of HBV replication (10). The secreted hepatitis B e-antigen, which has a structure similar to that of HBc, may interact with $\mathrm{HBc}$ monomers and form aberrant capsids that do not support the pgRNA package (10).

$\mathrm{HBc}$, which forms the icosahedral shell of capsids, consists of 183 or 185 amino acid residues (aa), depending on genotype (11). The primary structure of the core protein can be divided into two domains, namely, the N-terminal, which consists of 149 or 151 aa, depending upon the genotype, and directs HBc self-assembly $(12,13)$; the C-terminal constitutes 34 aa and is rich in arginine residues, essential for capsid formation. The deletion of the C-terminal domain inhibits pgRNA encapsidation (14). Capsid assembly consists of two steps, in which $\mathrm{HBc}$ monomers initially associate to yield a dimer intermediate via an intradimer interface (15). Numerous 
dimers subsequently form an intact capsid via a dimer-dimer interface. Whether higher-order HBc oligomers exist remains controversial (16). Several studies have proposed that aa 113-143 of HBc form the main dimer-dimer interface $(17,18)$.

In the present study, the aim was to identify a panel of residues located at the dimer-dimer interface based on the $\mathrm{HBc}$ crystal structure, and to investigate their effect on capsid function and viral replication. The results demonstrated that the $\mathrm{HBc}$ dimer-dimer interface was required for capsid assembly and viral replication. Targeting the dimer-dimer interface may be a novel and powerful antiviral strategy.

\section{Materials and methods}

$H B c$ crystal structure. The crystal structure of $\mathrm{HBc}$ was downloaded from the PDB (1QGT, https://www.rcsb. org/pdb/home/home.do). The dimer-dimer interface domains were visualized and mapped using Swiss-PdbViewer v4.0 software (http://www.expasy.org/spdbv/).

Plasmids. The 1.2-length (3,215 bp) HBV adw genome (GenBank accession no. AY518556; https://www.ncbi.nlm. nih.gov/genbank/) was obtained from the pHBV1.2 plasmid and inserted into pUC18 vector (Thermo Fisher Scientific, Inc., Waltham, MA, USA) under the control of the lac promoter, and was employed in the present study. Plasmid pHBV1.2-core- was derived from pHBV1.2 by introducing a stop codon (TAT $\rightarrow$ TAG) into the $\mathrm{C}$ gene at the Y38 position and ligating to pUC18 vector by HindIII and PshAI digestion, thereby preventing $\mathrm{HBc}$ production. In addition, the plasmid 1-183 flag, which directs the expression of the HBc gene under the control of the cytomegalovirus promoter, was generated using $X b a I$ and EcoRI enzymes, the pcDNA3.1 vector, along with a FLAG tag (N-DYKDDDDK-C) fused to the C-terminus.

Site-directed mutagenesis. All HBc mutant plasmids (pHBc14-18M, pHBc120-135M, pHBc23-39M and $\mathrm{pHBc} 122-139 \mathrm{M})$ were derived from the 1-183 flag plasmid. The altered residues of each domain were presented in Table I. Acidic amino acids were mutated into basic amino acids and vice versa, and amino acids with long side chains were mutated into glycine, to abolish the hydrophobic effect of the four domains. All point mutations generated within the core protein expression vector 1-183 flag were similarly generated according to a previously described polymerase chain reaction (PCR)-based method and the plasmid sequences were analyzed with BioEdit version 7.0.5 (http://www.mbio.ncsu.edu/BioEdit/page2.html) (19). PCR reactions were performed using Invitrogen ${ }^{\mathrm{TM}}$ PCR SuperMix (Thermo Fisher Scientific, Inc.) according to the manufacturer's instructions. In order to establish the 1-183 flag plasmid, a template pHBV1.2 plasmid, and 1-183 flag forward (For) and 1-183 flag reverse (Rev) primers were used. The $\mathrm{pHBC} 14-18$ plasmid was established using 1-183 flag as a template, and aa14-18 For and 1-183 flag Rev primers. pHBc23-39M, pHBc120-135M and pHBc122-139M plasmids were established via two cycles of PCR. For example, for pHBc120-135M plasmids, aa120-135 For and 1-183 flag Rev, and 1-183 flag For, and aa120-135 Rev primers were used for the first round of PCR, and pHBV1.2 was used as a template; the two products from the first round of PCR were subsequently mixed and annealed at $72^{\circ} \mathrm{C}$, and used as the template for the second round of PCR, where 1-183 flag For and 1-183 flag Rev primers were used. All PCR products were cloned into pcDNA3.1 vectors (Thermo Fisher Scientific, Inc., Waltham, MA, USA) using $X b a \mathrm{I}$ and EcoRI enzymes for digestion and ligation, respectively. The pHBV1.2-core ${ }^{-}$was generated using the aforementioned method but instead using HindIII For and Y38 Rev, and Y38 For and PshAI Rev primers for the first round of PCR; the two products from the first round of PCR were annealed and used as a template for the second round of PCR, where HindIII For and PshAI Rev primers were used. The PCR products were introduced to pUC18 vector by HindIII and PshAI digestion and ligation.

Cell culture and transfection. Human hepatoma HepG2 cells (American Type Culture Collection, Manassas, VA, USA) were cultured in Dulbecco's modified Eagle's medium (DMEM)-F12 (Invitrogen; Thermo Fisher Scientific, Inc.) containing $10 \%$ (v/v) fetal bovine serum (Gibco; Thermo Fisher Scientific, Inc.) at $37^{\circ} \mathrm{C}$ under $5 \% \mathrm{CO}_{2}$. HepG2 cells were co-transfected with $\mathrm{HBc}$ mutant plasmids and wild-type (WT) pHBV1.2. 1-183 flag and pcDNA3.1 were also transfected into HepG2 cells to establish control groups. All transfections were conducted using FuGENE HD transfection reagent (Promega Corporation, Madison, WI, USA) according to the manufacturer's protocols. The day prior to transfection, $5 \times 10^{5}$ cells were seeded into a 6 -well plate and cultured at $37^{\circ} \mathrm{C}$. After $20 \mathrm{~h}, 6 \mu \mathrm{l}$ FuGENE HD reagent and $2 \mu \mathrm{g} \mathrm{HBc}$ mutant plasmid or different amounts of $\mathrm{HBc}$ mutant plasmids (0.2-3.2 $\mu \mathrm{g})$ together with $1 \mu \mathrm{g}$ pHBV1.2 were used for transfection. According to preliminary experiments using a pCMV-GFP plasmid, 35\% of target HepG2 cells were successfully transfected. Five days post-transfection, cells were harvested for further analysis.

Viral particle assays. The secreted virion and intracellular capsid were assayed as previously described with some modifications (20). Briefly, after 5 days post-transfection, $1 \mathrm{ml}$ cell culture DMEM-F12 was harvested from the 6-well plate and secreted HBV virion was precipitated by adding dry PEG 8000 (Sigma-Aldrich; Merck KGaA, Darmstadt, Germany) to a final concentration of $10 \%(\mathrm{w} / \mathrm{v})$, and then incubated at $4^{\circ} \mathrm{C}$ for $1 \mathrm{~h}$. The precipitate was collected by centrifugation $(1,000 \mathrm{x} \mathrm{g}, 20 \mathrm{~min}$ at $4^{\circ} \mathrm{C}$ ) and dissolved in $40 \mu \mathrm{l}$ DMEM-F12. For the extraction of the intracellular capsid, cells from each well were washed three times with PBS and resuspended in $500 \mu 1$ lysis buffer [150 mM NaCl, $50 \mathrm{mM}$ Tris- $\mathrm{HCl}$ (pH 7.5), $5 \mathrm{mM} \mathrm{MgCl}_{2}, 0.2 \%$ (v/v) Nonidet P-40] on ice for $10 \mathrm{~min}$. The supernatant of the cell lysate was collected by centrifugation at $12,000 \mathrm{x}$ g for $10 \mathrm{~min}$ at $4^{\circ} \mathrm{C}$, precipitated with PEG 8000 (final concentration 10\%, w/v) for $1 \mathrm{~h}$ at $4^{\circ} \mathrm{C}$, and then dissolved in $60 \mu \mathrm{l}$ DMEM-F12. A total of $10 \mu \mathrm{l}$ secreted virions and intracellular capsids were fractionated via electrophoresis on a non-denaturing $1 \%$ agarose gel and transferred onto a nitrocellulose membrane overnight by blotting with 20X SSC buffer [ $3 \mathrm{M} \mathrm{NaCl}, 300 \mathrm{mM}$ sodium citrate ( $\mathrm{pH} 7)]$. To detect the secreted virions and intracellular capsids, the membrane was blocked at room temperature for $1 \mathrm{~h}$ with Tris-buffered saline supplemented with 5\% non-fat dry milk and $0.2 \%$ Tween-20. Subsequently, the virions were probed with monoclonal anti-envelope antibodies (1:2,000; cat. no. 18-0023; Invitrogen; Thermo Fisher Scientific, Inc.) or with a polyclonal antibody against core antigen (1:1,000; cat. no. B0586; Dako; 
Table I. HBc mutants of the dimer-dimer interface.

\begin{tabular}{lll}
\hline $\mathrm{HBc}$ & \multicolumn{1}{c}{ Wild-type } & \multicolumn{1}{c}{ Mutant } \\
\hline $14-18$ & ELLSF & KLLSG \\
$120-135$ & VSFGVWIRTPPAYRPP & GSFGGWIDTGPAARGG \\
$23-39$ & FFPSIRDLLDTASALYR & GFGSIRKLLDTASAGYD \\
$122-139$ & FGVWIRTPPAYRPPNAPI & GGVWIRTPPAYRPPNAPG \\
\hline
\end{tabular}

The corresponding residues of mutation are marked in bold. $\mathrm{HBc}$, hepatitis B virus core.

Agilent Technologies, Inc., Santa Clara, CA, USA) for $3 \mathrm{~h}$ at room temperature. Bound antibodies were detected using horseradish peroxidase-labeled secondary antibodies for $3 \mathrm{~h}$ at room temperature (goat anti-mouse; 1:5,000; cat. no. RM3001 and goat anti-rabbit; 1:4,000; cat. no. RM3002; both Beijing Ray Antibody Biotech, Beijing, China; http://www.rayantibody. $\mathrm{com} /$ ). Proteins were visualized using an ECL Plus chemiluminescence kit (Amersham Pharmacia Biotech; GE Healthcare, Chicago, IL, USA). Densitometry was performed using Quantity One ${ }^{\circledR}$ software version 4.6.3 (Bio-Rad Laboratories, Inc., Hercules, CA, USA).

SDS-PAGE and western blotting. Five days post-transfection, cells were treated with lysis buffer. Protein concentration was determined using the Pierce ${ }^{\mathrm{TM}}$ BCA Protein Assay kit (Thermo Fisher Scientific, Inc.) and adjusted to $5 \mu \mathrm{g} / \mu \mathrm{l}$ with lysis buffer. A total of $50 \mu \mathrm{g} / 10 \mu \mathrm{l}$ cell lysate was separated by $12 \%$ SDS-PAGE and transferred onto a polyvinylidene fluoride membrane. The membrane was blocked with $5 \%$ non-fat milk in Tris-buffered saline for $0.5 \mathrm{~h}$ at room temperature, and incubated with primary anti-flag (1:2,000; cat. no. PM020; MBL International Co., Woburn, MA, USA), polyclonal rabbit anti-core $(1: 1,000$; cat. no. B0586; Dako; Agilent Technologies, Inc.) and monoclonal mouse anti-GAPDH (1:3,000; cat. no. RM 2002; Beijing Ray Antibody Biotech) antibodies for $2 \mathrm{~h}$ at room temperature. Bound antibodies were detected with horseradish peroxidase-labeled secondary antibodies for $3 \mathrm{~h}$ at room temperature (goat anti-rabbit; 1:4,000; cat. no. RM3002 and goat anti-mouse; 1:5,000; cat. no. RM3001; both Beijing Ray Antibody Biotech) and visualized using an ECL Plus chemiluminescence kit (Amersham Pharmacia Biotech; GE Healthcare). The density of each band was analyzed using Quantity One software version 4.6.3 (Bio-Rad Laboratories, Inc.).

Southern blotting. To detect capsid-associated HBV DNA, a digoxigenin (DIG)-labeled double-stranded specific full-length HBV probe was synthesized from $\mathrm{pHBV} 1.2$ with a PCR DIG Probe Synthesis Kit (Roche Diagnostics, Indianapolis, IN, USA) according to the manufacturer's protocols. Primers (P1, 5'-CCG GAAAGCTTGAGCTCTTCTTTTTCACCTCTGCCTAAT CA-3' and P2, 5'-CCGGAAAGCTTGAGCTCTTCAAAA AGTTGCATGGTGCTGG-3') were used to synthesize the HBV probe. Extraction of intracellular capsid-associated HBV DNA and Southern blot analysis were conducted as previously described (21) using a DIG DNA Labeling and Detection Kit (Sigma-Aldrich; Merck KGaA, Darmstadt, Germany). In brief, $15 \mu \mathrm{g}$ of capsid-associated HBV DNA per lane was electrophoresed on a $1 \%$ agarose gel. Nucleic acids were then transferred onto a positively charged nylon membrane (GE Healthcare). These blots were hybridized with a DIG-labeled double-stranded specific full-length HBV probe overnight at room temperature.

Quantification of HBV DNA and cccDNA using quantitative (q) $P C R$. The nuclear cccDNA and virion HBV DNA in the culture medium were quantified by qPCR on a LightCycler $^{\circledR} 480$ system (Roche Diagnostics, Indianapolis, IN, USA). Total DNA was extracted using the QIAamp DNA Mini kit (Qiagen $\mathrm{GmbH}$, Hilden, Germany) according to the manufacturer's instructions. Isolated DNA was treated with 10,000 U/ml T5 exonuclease (New England BioLabs, Inc., Ipswich, MA, USA) for $30 \mathrm{~min}$ at $37^{\circ} \mathrm{C}$ in $10 \mu \mathrm{l}$ reaction volume followed by heat inactivation at $95^{\circ} \mathrm{C}$ for $5 \mathrm{~min}$ and 4 -fold dilution with distilled water. Two different primer and TaqMan probe sets were synthesized by Sangon Biotech Co., Ltd. (Shanghai, China) for the detection of HBV-DNA and HBV cccDNA in the culture medium. The primers and probe design were according to a previous study (22). The PCR primers for the amplification of cccDNA were: For, 5'-GGGGCGCACCTCTCTTTA-3' and Rev, 5'-AGG CACAGCTTGGAGGC-3'. The TaqMan hydrolysis probe for the detection of the amplified 367 bp DNA fragment was as follows: 5'-FAM-TTCTCATCTGCCGGACCGTG-BHQ-3'. The PCR primers for the amplification of total DNA were: For, 5'-GCCAAAATTCGCAGTCC-3' and Rev, 5'-AAACTG AGCCAGGAGAAA-3'. The Taqman hydrolysis probe for the detection of the amplified $376 \mathrm{bp}$ DNA fragment was as follows: 5'-FAM-TTCCTCTTCATCCTGCTGCTATGCC-BHQ-3'. The amplification program consisted of an initial denaturing step at $94^{\circ} \mathrm{C}$ for $10 \mathrm{~min}$, followed by 45 cycles of $95^{\circ} \mathrm{C}$ for $10 \mathrm{sec}, 55^{\circ} \mathrm{C}$ for $20 \mathrm{sec}$ and $72^{\circ} \mathrm{C}$ for $20 \mathrm{sec}$.

The pHBV1.2 plasmid, which contained 1.2 copies of the HBV genome, was extracted using the QIAGEN Plasmid Mini kit (Qiagen $\mathrm{GmbH}$ ), according to the manufacturer's instructions. The plasmid concentration $(\mathrm{mg} / \mathrm{ml})$ was measured at $260 \mathrm{~nm}$ using a spectrophotometer. The copy number of the plasmid was calculated using the following formula: Copies $/ \mathrm{ml}=\mathrm{C} / \mathrm{M} \times \mathrm{A}$ (where $\mathrm{C}$ is the plasmid concentration, $\mathrm{M}$ is the molecular weight and $\mathrm{A}$ is the Avogadro constant). Ten-fold serial dilutions of pHBV1.2 plasmid stock $\left(10^{9}-10^{2}\right.$ copies $\left./ \mathrm{ml}\right)$ were used to establish qPCR standard curves for detecting HBV cccDNA and HBV total DNA. The primers and probe are the same as when the samples were tested. Each experiment was performed in triplicate.

RNA extraction and northern blotting. Total cellular RNA was extracted from transfected cells with TRIzol reagent (Invitrogen; Thermo Fisher Scientific., Inc.). Total RNA (5 $\mu \mathrm{g})$ 
per lane was separated on a $1 \%$ agarose gel containing $2.2 \mathrm{M}$ formaldehyde and transferred onto a positively charged nylon membrane (GE Healthcare) with 20X SSC buffer. The probe and hybridization procedure was conducted in accordance with that for Southern blot analysis.

Conservation analysis of capsid residues. A total of 9,386 $\mathrm{HBc}$ sequences were downloaded from HBVdb (https://hbvdb.ibcp. fr/HBVdb/HBVdbIndex) (23) and submitted to Clustal Omega (https://www.ebi.ac.uk/Tools/msa/clustalo/; version 1.2.4) for alignment (24).

Statistical analysis. SPSS 16.0 software (SPSS, Inc., Chicago, IL, USA) was employed to analyze the experimental data, which are presented as the mean \pm standard error of the mean. A Mann-Whitney U test was performed to compare the levels of viruses between $\mathrm{HBc}$ mutant and control groups. $\mathrm{P}<0.05$ was considered to indicate a statistically significant difference. All experiments were performed in triplicate.

\section{Results}

Identification of $\mathrm{HBc}$ dimer-dimer interface. The crystal structure of $\mathrm{HBc}$ has been reported previously (18). $\mathrm{HBc}$ is mainly a helical protein; the hydrophobic effect is the main force that directs its assembly (Fig. 1A). HBc monomers form a dimer via the intradimer interface to initiate assembly, and then several dimers are assembled to form an icosahedral capsid via the dimer-dimer interface (15). Based on its crystal structure, 16 aa located at the dimer-dimer interface were identified using Swiss-PdbViewer v4.0: i) 14E, 18F, 120V, 124V, 127R, 129P, 132Y, 134P and 135P; and ii) 23F, 25P, 29D, 37L, 39R, 122F, and 139I (Fig. 1B). These residues were classified into four domains based on the HBc primary sequences and spatial structure as follows: HBc14-18, HBc23-39, HBc120-135, and HBc122-139 (Table I). To determine the role of each of these domains in capsid assembly and function, all 16 residues were mutated in accordance with the following principles to abolish the hydrophobic effect of the four domains: Acidic amino acids were mutated into basic amino acids and vice versa, and amino acids with long side chains were mutated into glycine. Of note, two mutants with similar primary structures, HBc120-135 and HBc122-139, were generated in the present study as the residues were located within different dimer units in the HBc crystal structure. The two domains were presented in Fig. 1B as green and blue; the map of the plasmids employed in the present study were presented in Fig. 1C.

The HBV sequence alignment results demonstrated that residues $14 \mathrm{E}$ and $18 \mathrm{~F}$ are relatively conserved, whereas 29D and 132Y are highly conserved, and 25P and 139I are completely conserved across all 9,386 sequences (Fig. 2).

Capsid-like structure formed by HBc mutants. To determine whether all HepG2 cells were successfully transfected with $\mathrm{HBc}$ mutants, a FLAG tag was fused to the C-terminus of WT HBc (1-183 flag) and all mutant plasmids (Fig. 1C). After 5 days post-transfection, an anti-FLAG antibody was used in western blotting to detect its expression. A $21 \mathrm{kDa}$ band was observed in all mutant and 1-183 flag-transfected groups, indicating that all $\mathrm{HBc}$ mutants exhibited normal expression (Fig. 3A).
To determine whether mutations of the dimer-dimer interface affect capsid formation, HepG2 cells were co-transfected with each $\mathrm{HBc}$ mutant and $\mathrm{pHBV} 1$.2-core ${ }^{-}$. A total of 5 days post-transfection, the intracellular capsid was isolated and analyzed on an agarose gel (native condition) or subjected to SDS-PAGE (denaturing and reducing conditions), and then detected with an anti-HBc antibody (Fig. 3B). The major epitope of the antibody is at the tip of the spike on the capsid surface (aa 78-83), which appeared to remain unaltered in the mutants. In the capsid structure, the spike epitope is exposed and accessible to the anti-HBc antibody (18). Following SDS-PAGE, 1-183 flag and all mutants were detected with the anti-core antibody, which indicated that the plasmids were successfully generated; however, under native conditions, $\mathrm{pHBc} 23-39 \mathrm{M}$ was not detected via an anti-HBc antibody, suggesting that the spike epitope was not properly exposed, thereby preventing the formation of capsid-like structures. Conversely, the three mutants, pHBc14-18M, pHBc120-135M and $\mathrm{pHBc} 122-139 \mathrm{M}$, assembled a capsid-like structure that could be detected with the corresponding antibody under native conditions (Fig. 3B).

As some of the mutants assembled capsid-like structures, whether these supported normal capsid function was investigated in the present study. To address this issue, each $\mathrm{HBc}$ mutant was respectively co-transfected into cells with pHBV1.2-core ${ }^{-}$, and the capsid-associated $3.5 \mathrm{~kb}$ pgRNA and HBV DNA were isolated 5 days post-transfection and detected with a specific HBV probe via northern and Southern blotting. None of the $\mathrm{HBc}$ mutants supported the assembly of the pgRNA package or viral replication (Fig. 3C and D). These findings indicated that these residues at the dimer-dimer interface are critical for capsid structure and function. Mutations within these regions may completely disrupt HBV replication.

Effect of HBc mutants on WT viral replication. As all the mutations were situated within the dimer-dimer interface, the mutants should remain to possess intact intradimer interfaces. The present study hypothesized that these interact with the WT $\mathrm{HBc}$ monomer to form aberrant dimers, which in turn may affect WT virus capsid assembly and function (Fig. 1A, lower panel). To validate this hypothesis, HepG2 cells were co-transfected with an equal amount of $\mathrm{HBc}$ mutant plasmid and WT pHBV1.2; after 5 days post-transfection, capsid-associated HBV DNA was extracted and identified by Southern blot analysis (Fig. 4A). The results demonstrated that pHBc14-18M significantly inhibited WT viral replication as presented by a $36.8 \%$ reduction in the expression levels of capsid-associated HBV DNA compared with in the 1-183 flag control; pHBc120-135M and $\mathrm{pHBc} 122-139 \mathrm{M}$ significantly inhibited HBV DNA production by 62.4 and $49.3 \%$, respectively, compared with the control (Fig. 4B). However, pHBc23-39M did not exhibit an inhibitory effect on WT viral replication. The present study proposed that pHBc23-39M could not form a capsid by itself, indicating that mutations within this domain may also inhibit $\mathrm{HBc}$ monomer interactions. Thus, $\mathrm{pHBc} 23-39 \mathrm{M}$ may not interact with WT $\mathrm{HBc}$ to affect capsid function and viral replication.

In addition, whether the mutants affect cccDNA and virion-associated HBV DNA in the medium was investigated in the present study. After 5 days post-transfection, the nuclear cccDNA and HBV DNA in the medium were isolated and quantitated using qPCR. The results revealed that 
A HBc monomer

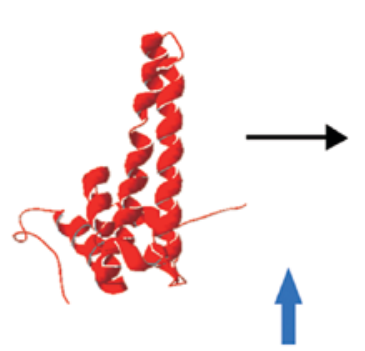

Intradimer interface

$\checkmark$
HBc dimer

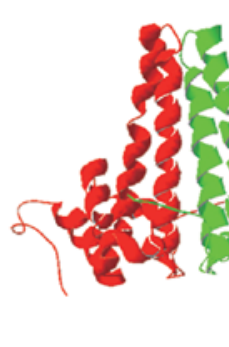

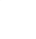

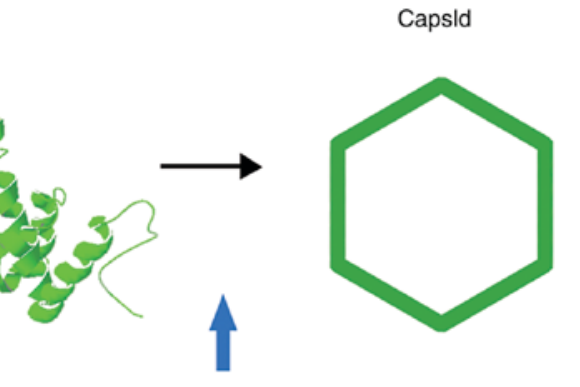

Dimer-dimer interface
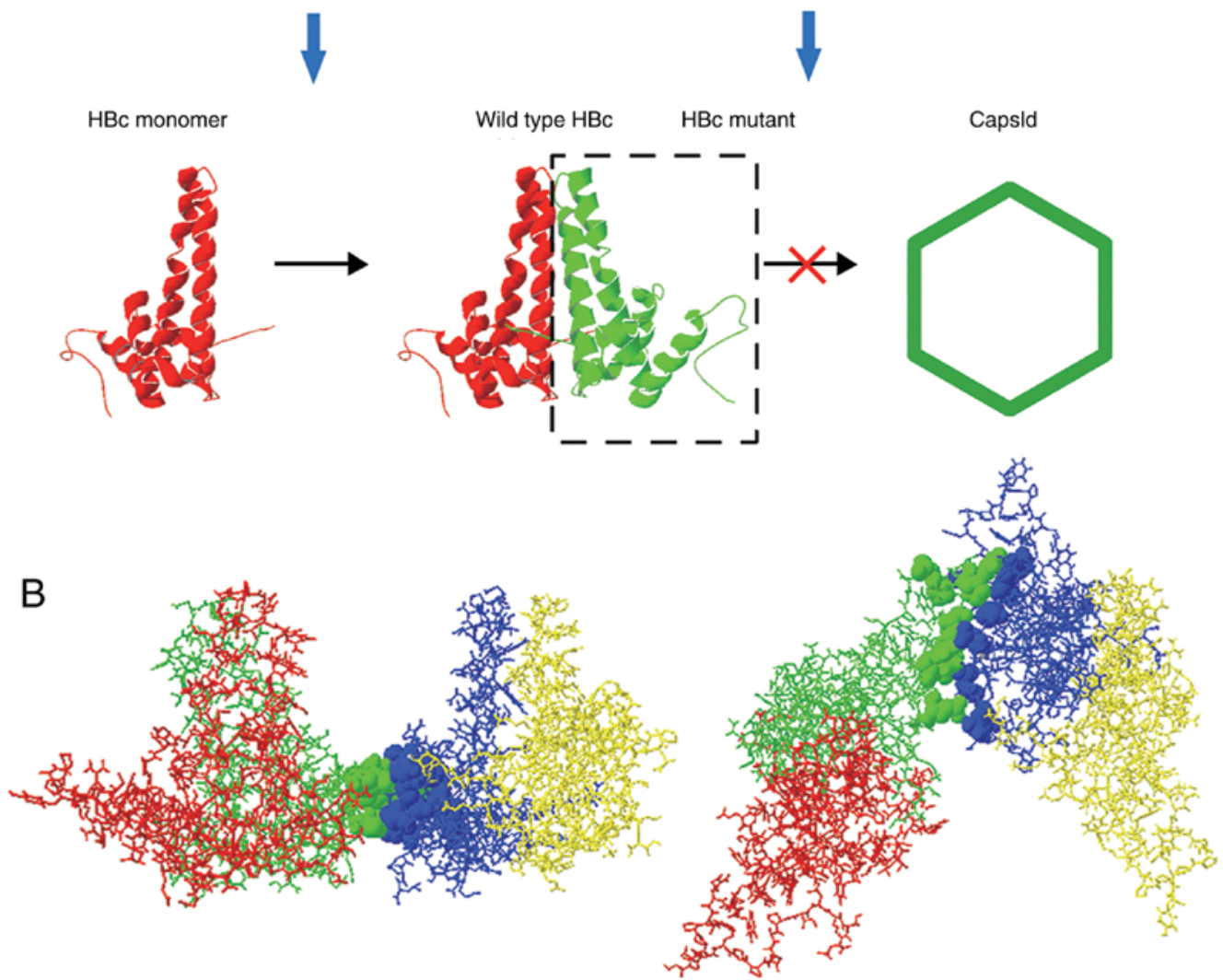

C

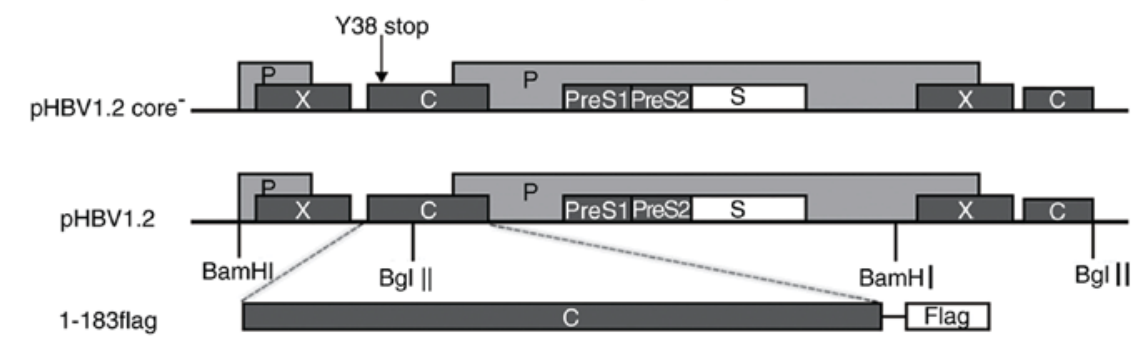

HBc mutants 14-18 23-39

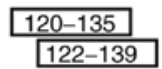

Figure 1. HBV capsid assembly and dimer-dimer interface. (A) Capsid assembly procedure. Upper panel, two $\alpha$-helical HBc monomers form a dimer via the intradimer interface, and numerous dimers subsequently form an icosahedral capsid via the dimer-dimer interface. Lower panel, $\mathrm{HBc}$ with mutations at the dimer-dimer interface may interact with WT HBc monomer via the intradimer interface to form an aberrant dimer, but disrupts the capsid structure or function. (B) Orthogonal perspectives of HBc (1QGT, Protein Data Bank) viewed normal to the local 2-fold axis (right) and along the 2-fold axis from the outside of the $\mathrm{HBc}$ (left); $4 \mathrm{HBc}$ monomer units are indicated by four different colors (red, green, blue and yellow). Residues at the dimer-dimer interface are presented as ribbons, indicated in green $(14 \mathrm{E}, 18 \mathrm{~F}, 120 \mathrm{~V}, 124 \mathrm{~V}, 127 \mathrm{R}, 129 \mathrm{P}, 132 \mathrm{Y}, 134 \mathrm{P}$ and $135 \mathrm{P})$ and blue $(23 \mathrm{~F}, 25 \mathrm{P}, 29 \mathrm{D}, 37 \mathrm{~L}, 39 \mathrm{R}, 122 \mathrm{~F}$ and $139 \mathrm{I})$, respectively. (C) Map of plasmids. For the expression of the HBV genome, 1.2 units of the HBV genome was inserted at the 3' end of the lac promoter in pUC18 to construct the pHBV1.2 plasmid. The open reading frames of the viral C, P, E and X genes are indicated by dark grey boxes. The C gene of pHBV1.2core- contained a point mutation of a stop codon at codon 38 (arrow). For the expression of WT and mutant HBc, the pcDNA3.1 vector was used. A FLAG tag was fused to the C-terminus of $\mathrm{HBc}$. Each $\mathrm{HBc}$ mutant is indicated by a white box. $\mathrm{HBV}$, hepatitis B virus; $\mathrm{HBc}, \mathrm{HBV}$ core protein; WT, wild-type.

HBc mutants exhibited no significant effect on the levels of cccDNA; however, the number of viruses in the medium of the
pHBc14-18M, pHBc120-135M, and pHBc122-139M groups was significantly decreased compared with in the 1-183 flag 


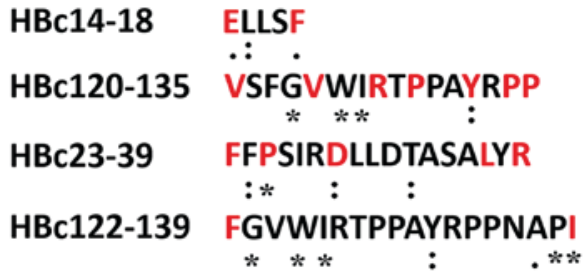

Figure 2. Conservation analysis of $\mathrm{HBc}$ sequences. A total of 9,386 $\mathrm{HBc}$ sequences were downloaded from the HBVdb database and analyzed with the Clustal Omega sequence aligning program. As the hydrophobic effect is the main force that directs capsid assembly, acidic residues, basic residues and those with long side chains were mutated (labeled in red). '? Denotes relatively conserved; ':' denotes highly conserved and '*' denotes completely conserved across 9,386 sequences. HBc, hepatitis B virus core protein.

control, which coincided with alterations in capsid-associated HBV DNA (Fig. 4C).

Effect of HBc mutants on WT virion production. In addition, how the HBc mutants affect WT virion production was investigated. After 5 days post co-transfection, virions in the media were collected and detected using an anti-envelope antibody. Since it was confirmed that $\mathrm{pHBc} 23-39 \mathrm{M}$ could not form capsids by itself or inhibit WT HBV replication, its effect on HBV virion production was not analyzed. The results revealed that pHBc14-18M,pHBc120-135M and pHBc122-139M significantly inhibited WT virion production by $>50 \%$ compared with in the 1-183 flag control (Fig. 5; $\mathrm{P}<0.05$ ). pHBc14-18M significantly decreased capsid-associated HBV DNA by $36.8 \%$ compared with the 1-183 flag control (Fig. 4B; $\mathrm{P}<0.05)$, indicating that this domain may also participate in the late events of the HBV life cycle and virion formation.

$H B c$ mutants inhibit WT replication and virion production in a dose-dependent manner. To further confirm the effect of $\mathrm{HBc}$ dimer-dimer mutations on WT replication and virion production, different amounts of $\mathrm{HBc}$ mutant plasmids (0.2-3.2 $\mu \mathrm{g}$ ) together with $1 \mu \mathrm{g}$ pHBV1.2 were transfected into HepG2 cells. After 5 days post-transfection, the production of HBV DNA and virions in the culture medium was detected as aforementioned (Fig. 6). HBc mutants, including pHBc14-18M, pHBc120-135M and pHBc122-139M significantly inhibited HBV DNA and virion production in a dose-dependent manner (Fig. 6; P<0.05). pHBc120-135 exhibited the most potent antiviral effect, as it inhibited WT replication and virion production by 84 and $83.1 \%$, when co-transfected with a plasmid ratio of 3.2:1 and 1.6:1 $\mu \mathrm{g}$, respectively.

\section{Discussion}

The aim of the present study was to identify the role of the $\mathrm{HBc}$ dimer-dimer interface in capsid assembly and function.
A

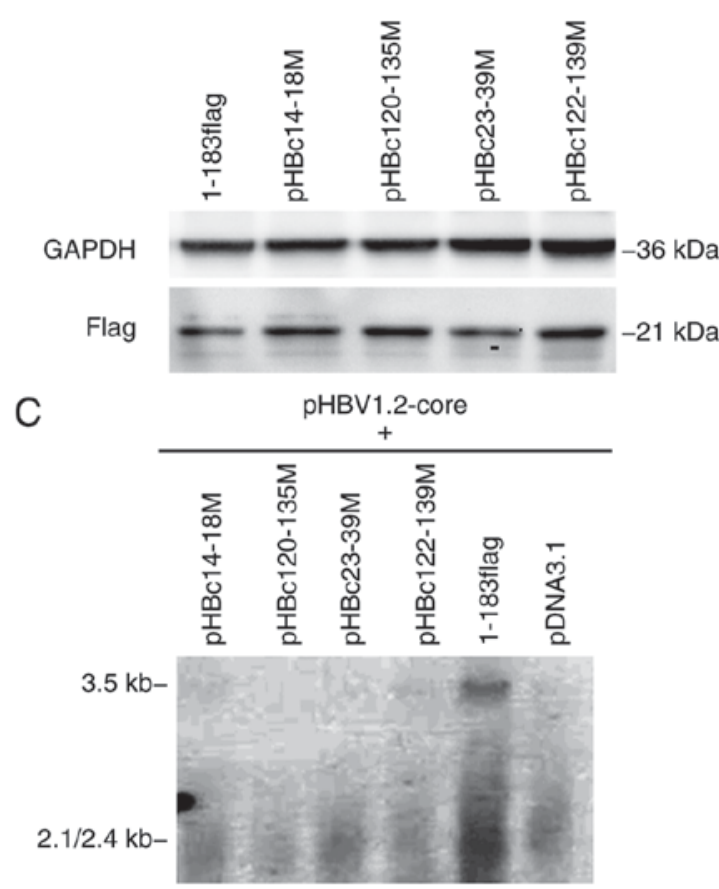

B

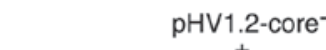

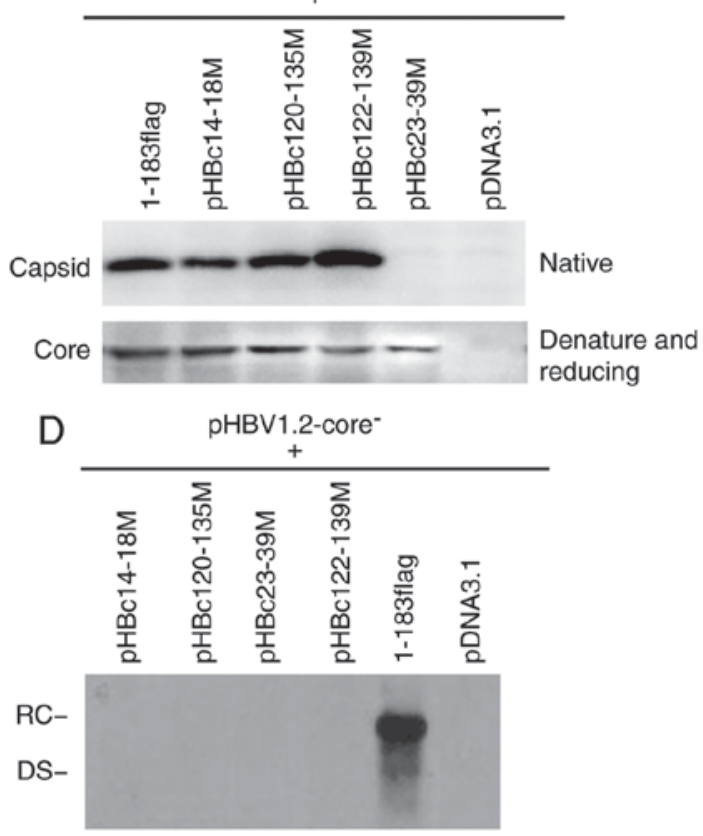

Figure 3. Capsid formed by HBc with dimer-dimer mutations. (A) HepG2 cells were transfected with $2 \mu \mathrm{g}$ HBc mutant or 1-183 flag (control) using FuGENE HD transfection reagent according to the manufacturer's instructions. After 5 days post-transfection, cells lysates were isolated, resolved with SDS-PAGE and probed with anti-GAPDH or -flag antibody. (B) HepG2 cells were co-transfected with an equal amount of HBc mutant and pHBV1.2core; 5 days post-transfection, cell lysates were analyzed on a native agarose gel (for capsid detection) or subjected to SDS-PAGE (for core protein detection), and then detected with a polyclonal antibody against the core antigen. The major epitope of the antibody is at the tip of the spike on the capsid surface (amino acid residues 78-83), which were unaltered in mutants. Under native conditions, the spike epitope emerging from the capsid should be exposed and accessible to the anti-HBc antibody.

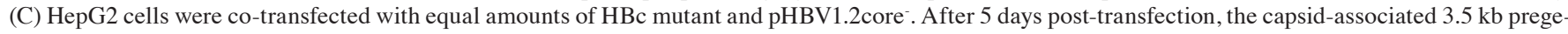
nomic RNA was detected with a specific HBV DNA probe. The positive and negative controls were 1-183 flag and pcDNA3.1, respectively. (D) After 5 days post co-transfection, capsid-associated HBV DNA was purified and subjected to Southern blot analysis with a specific HBV DNA probe. HBV, hepatitis B virus; HBc, HBV core protein; DS, double-stranded DNA; RC, relaxed circular DNA. 
A

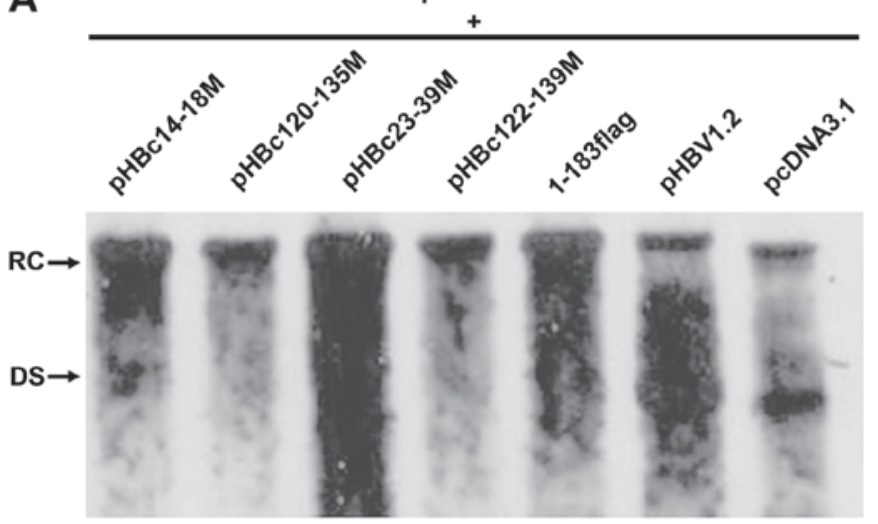

C

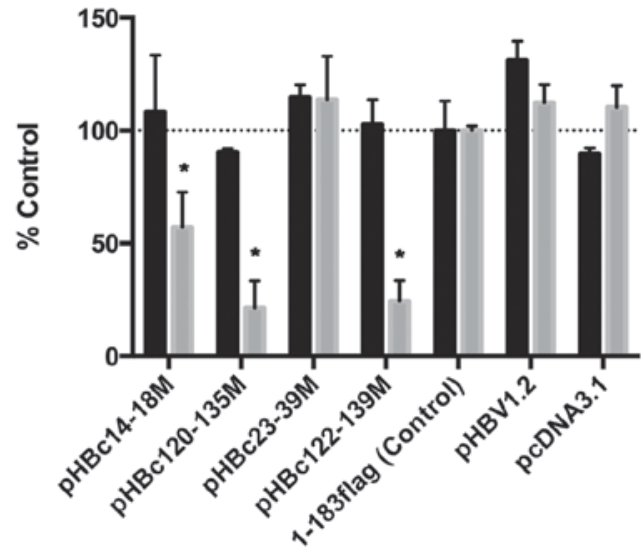

B Capsid related HBV DNA

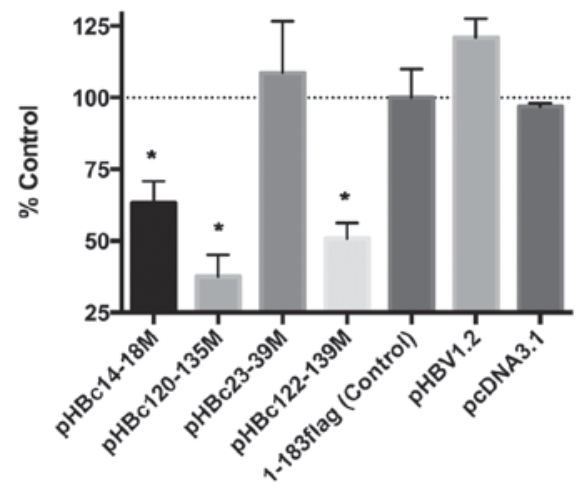

cccDNA

Medium HBV DNA

Figure 4. HBc dimer-dimer mutants inhibit WT replication. (A) HepG2 cells were co-transfected with an equal amount of HBc mutant and pHBV1.2. After 5 days post-transfection, the capsid-associated HBV DNA was isolated and detected with a specific HBV DNA probe. (B) Relative density of the RC and DS bands of each group in (A) was analyzed using Quantity One software (version 4.6.3). The experiment was repeated in triplicate. Date are presented as the mean \pm standard error of the mean. "P<0.05 vs. 1-183 flag control group. (C) After 5 days post co-transfection, the cell medium was collected and then purified with a $0.45-\mu \mathrm{m}$ filter; the nuclear cccDNA was also purified. Nuclear cccDNA and virion HBV DNA in the culture medium were quantified by qPCR on a LightCycler $^{\circledR} 480$ system. The experiment was repeated in triplicate. Date are presented as the mean \pm standard error of the mean. ${ }^{*} \mathrm{P}<0.05$ vs. 1-183 flag control group. DS, double-stranded DNA; HBV, hepatitis B virus; HBc, HBV core protein; RC, relaxed circular DNA.

A

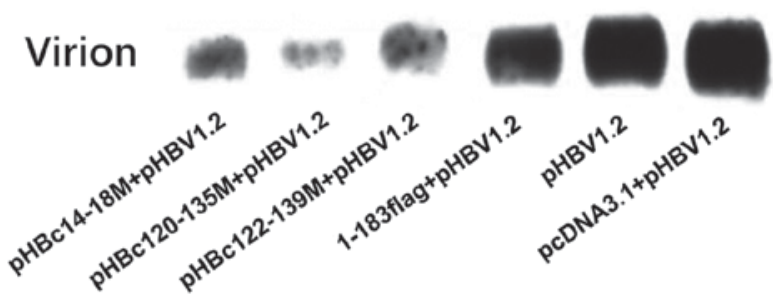

B

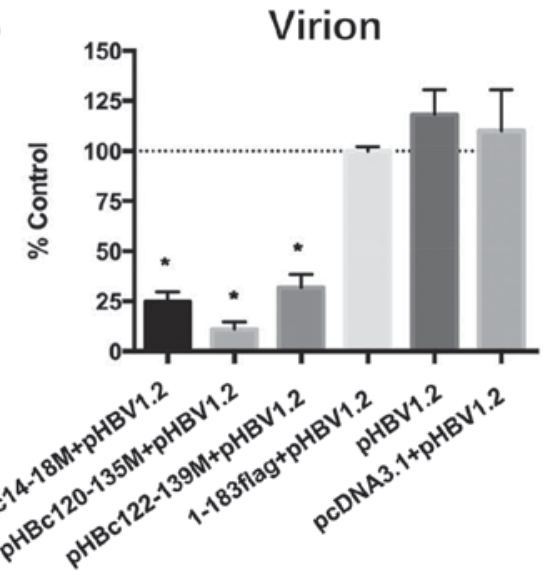

Figure 5. HBc dimer-dimer mutants inhibit virion production. (A) HepG2 cells were co-transfected with an equal amount of HBc mutant and pHBV1.2. After 5 days post-transfection, the virions were collected, purified with a $0.45-\mu \mathrm{m}$ filter, analyzed on a native agarose gel and then detected with an anti-HBsAg antibody. (B) Density of each band in (A) was analyzed using Quantity One software (version 4.6.3). The experiment was repeated in triplicate. Date are presented as the mean \pm standard error of the mean. ${ }^{~} \mathrm{P}<0.05$ vs. 1-183 flag control group. HBV, hepatitis B virus; HBc, HBV core protein; HBsAg, HBc antigen.

HBV consists of an outer lipidic envelope protein shell and inner icosahedral capsid, which is crucial for pgRNA packing, genome replication, and viral envelopment. A previous study demonstrated that capsid assembly is strongly associated with the presence of supplementary cccDNA (25). Dysregulation of capsid assembly could be a powerful tool for inhibiting viral replication. A variety of agents that inhibit capsid assembly, including intracellular single-chain antibody (26), peptide 
A

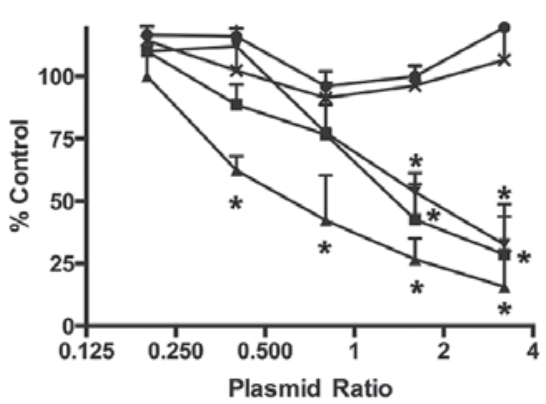

B

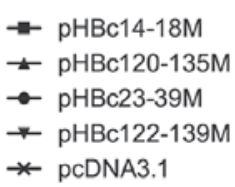

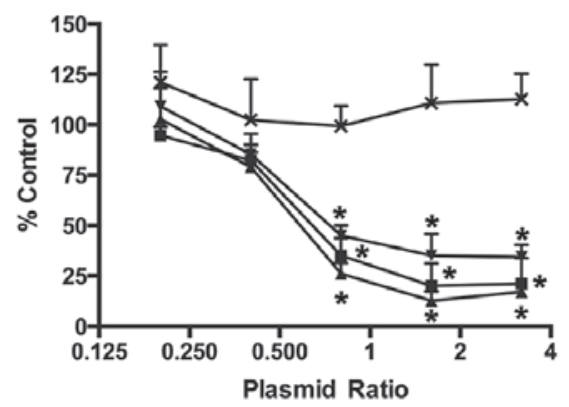

$\rightarrow \mathrm{pHBc} 14-18 \mathrm{M}$

$\pm \mathrm{pHBc} 120-135 \mathrm{M}$

$*$ pHBc122-139M

* pCDNA3.1

Figure 6. HBc dimer-dimer mutants inhibit capsid-associated HBV DNA and virion production in a dose-dependent manner. HepG2 cells were co-transfected with different ratios of $\mathrm{HBc}$ mutants $(0.2,0.4,0.8,1.6$ and $3.2 \mu \mathrm{g})$ to $\mathrm{pHBV} 1.2(1 \mu \mathrm{g})$. After 5 days post-transfection, the (A) capsid-associated HBV DNA and (B) virions in the medium were purified; the amounts produced were determined. The experiment was repeated in triplicate. Date are presented as the mean \pm standard error of the mean. ${ }^{*} \mathrm{P}<0.05$ vs. pcDNA3.1.

aptamers (27), N-nonyl-deoxy-galactonojirimycin (28), and bis-ANS (29) demonstrated potent antiviral effects in vitro and in vivo. In the present study, it was reported that some domains of the dimer-dimer interface are may be essential for capsid function and virus viability.

The successful identification of the HBc crystal structure provides insight to improve understanding of its assembly and function. In the present study, four domains of the $\mathrm{HBC}$ dimer-dimer interface were identified based on its crystal structure; two of the domains (HBc14-18 and HBc23-39) were located at the $\mathrm{N}$-terminus of $\mathrm{HBc}$, whereas the two other domains (HBc120-135 and HBc122-139) encompassed aa 113-143, which have been proposed to comprise the key dimer-dimer interaction site $(18,19)$. The present study demonstrated that mutations involving HBc23-39 completely disable capsid assembly, and pHBc23-39 could not interact with WT $\mathrm{HBc}$ to interfere with its replication. These findings indicated that HBc23-39 may be associated with the intradimer interface, which is critical for $\mathrm{HBc}$ monomer interactions. In addition, P25 and D29 of HBc23-39 are absolutely and highly conserved, respectively across $9,386 \mathrm{HBc}$ sequences. The mutation of these two residues may account for failure in capsid assembly. Conversely, pHBc14-18M formed a capsid-like structure, but it did not support pgRNA packaging or HBV DNA replication in the present study.

The S17 and F18 mutations, which were introduced in pHBc14-18M, allow capsid assembly but inhibit mature virion formation (20,21). Similarly, HBc120-135 and HBc122-139 formed capsid-like structures, but did not support replication. The V124 mutation only permitted the assembly of non-capsid polymers as observed by electron microscopy (30). Collectively, these findings suggested that the HBc dimer-dimer interface serves pivotal roles in maintaining normal capsid structure and function. Interestingly, $\mathrm{HBc} 23-39$ may be a potential antiviral target as minor alterations of its residues efficiently inhibited capsid formation.

As the approved nucleotide analogs could not eradicate nuclear cccDNA, the main source of persistent infection, further investigation and evaluation of novel antiviral agents is warranted. $\mathrm{HBc}$ is recruited to the cccDNA minichromosome in vivo and maintains the permissive epigenetic state in the critical region of cccDNA $(4,31)$. The present study demonstrated that $\mathrm{pHBc} 14-18 \mathrm{M}$, pHBc120-135M and
pHBc122-139M do not support viral replication. Furthermore, these mutants interacted with WT HBc, and inhibited viral replication and virion production. Therefore, these domains may be utilized as anti-HBc targets; once bound to small molecules, these domains are prevented from participating in capsid assembly and may also interfere with WT capsid formation and function (7).

In the present study, pHBc14-18M significantly inhibited HBV replication by $36.8 \%$, but effectively inhibited virion formation by $75.1 \%$. F18 of pHBc14-18M was reported to also inhibit particle envelopment (19) which may explain the observed differences in the effects on viral replication and virion production.

pHBc120-135M exhibited the most efficient antiviral effect. Y132 of pHBc120-135 is a highly conserved residue of $\mathrm{HBc}$ and is almost fully buried in the capsid crystal structure (18). Whether Y132 or other residues, or their combined synergistic effect is responsible for the potent antiviral effect of pHBc120-135M requires further study; the generation of a series of single point mutants may further elucidate these associations. In the present study pHBc122-139M contains only two mutated residues, F122 and I139; however, effective inhibition of WT viral replication was observed, indicating the importance of these two residues in capsid function. A single mutation of F122 has been reported to inhibit pgRNA packaging (20).

The present study also determined whether dimer-dimer mutations affect the quantity of nuclear cccDNA. No significant alterations in the quantity of cccDNA were observed after 5 days post-co-transfection. The results of the present study revealed that $\mathrm{HBc}$ dimer-dimer mutants inhibited WT replication in a dose-dependent manner, thereby excluding the possibility of nonspecific interaction between mutants and WT HBc.

Our study demonstrated that the HBc dimer-dimer interface is required for capsid assembly and viral replication. Targeting the dimer-dimer interface may be a novel and powerful antiviral strategy. The findings of the present study provide an improved understanding of the HBV life cycle and may contribute to the development of novel antiviral treatments.

\section{Acknowledgements}

Not applicable. 


\section{Funding}

The present study was supported by the Major Program of National Natural Science Foundation of China (grant no. 81500287), the Natural Science Foundation of Guangdong (grant no. 2016A030313357), the Science and technology Planning Project of Guangdong (grant nos. 2014A020212575 and 2016A020215215).

\section{Availability of data and materials}

The datasets used during the present study are available from the corresponding author on reasonable request.

\section{Authors' contributions}

KD and YZ conceived and designed the study. CLZ, YMF, ZXX, YZ and KD performed the experiments. CLZ, YZ and KD wrote the manuscript. All authors read and approved the manuscript.

\section{Ethics approval and consent to participate}

This study was approved by the Institutional Review Board of the Third Hospital of Sun Yat-Sen University (Guangzhou, China). Informed consent was obtained from all patients.

\section{Patient consent for publication}

Not applicable.

\section{Competing interests}

The authors declare that they have no competing interests.

\section{References}

1. Dienstag JL: Hepatitis B virus infection. N Engl J Med 359: 1486-1500, 2008.

2. Seeger $C$ and Mason WS: Molecular biology of hepatitis B virus infection. Virology 479-480: 672-686, 2015.

3. Rabe B, Vlachou A, Panté N, Helenius A and Kann M: Nuclear import of hepatitis B virus capsids and release of the viral genome. Proc Natl Acad Sci USA 100: 9849-9854, 2003.

4. Guo YH, Li YN, Zhao JR, Zhang J and Yan Z: HBc binds to the $\mathrm{CpG}$ islands of $\mathrm{HBV}$ cccDNA and promotes an epigenetic permissive state. Epigenetics 6: 720-726, 2011.

5. Seeger C and Mason WS: Hepatitis B virus biology. Microbiol Mol Biol Rev 64: 51-68, 2000.

6. Wu G, Liu B, Zhang Y, Li J, Arzumanyan A, Clayton MM, Schinazi RF, Wang Z, Goldmann S, Ren Q, et al: Preclinical characterization of GLS4, an inhibitor of hepatitis B virus core particle assembly. Antimicrob Agents Chemother 57: 5344-5354, 2013.

7. Bourne CR, Finn MG and Zlotnick A: Global structural changes in hepatitis B virus capsids induced by the assembly effector HAP1. J Virol 80: 11055-11061, 2006.

8. Stray SJ, Bourne CR, Punna S, Lewis WG, Finn MG and Zlotnick A: A heteroaryldihydropyrimidine activates and can misdirect hepatitis B virus capsid assembly. Proc Natl Acad Sci USA 102: 8138-8143, 2005.

9. Delaney WE IV, Edwards R, Colledge D, Shaw T, Furman P, Painter G and Locarnini S: Phenylpropenamide derivatives AT-61 and AT-130 inhibit replication of wild-type and lamivudine-resistant strains of hepatitis B virus in vitro. Antimicrob Agents Chemother 46: 3057-3060, 2002.

10. Scaglioni PP, Melegari M and Wands JR: Posttranscriptional regulation of hepatitis $\mathrm{B}$ virus replication by the precore protein. J Virol 71: 345-353, 1997.
11. Chain BM and Myers R: Variability and conservation in hepatitis B virus core protein. BMC Microbiol 5: 33, 2005.

12. Birnbaum F and Nassal M: Hepatitis B virus nucleocapsid assembly: Primary structure requirements in the core protein. J Virol 64: 3319-3330, 1990.

13. Zlotnick A, Cheng N, Conway JF, Booy FP, Steven AC, Stahl SJ and Wingfield PT: Dimorphism of hepatitis B virus capsids is strongly influenced by the C-terminus of the capsid protein. Biochemistry 35: 7412-7421, 1996

14. Gallina A, Bonelli F, Zentilin L, Rindi G, Muttini M and Milanesi G: A recombinant hepatitis B core antigen polypeptide with the protamine-like domain deleted self-assembles into capsid particles but fails to bind nucleic acids. J Virol 63: 4645-4652, 1989.

15. Zhou S and Standring DN: Hepatitis B virus capsid particles are assembled from core-protein dimer precursors. Proc Natl Acad Sci USA 89: 10046-10050, 1992.

16. Lingappa JR, Martin RL, Wong ML, Ganem D, Welch WJ and Lingappa VR: A eukaryotic cytosolic chaperonin is associated with a high molecular weight intermediate in the assembly of hepatitis B virus capsid, a multimeric particle. J Cell Biol 125: 99-111, 1994.

17. König S, Beterams G and Nassal M: Mapping of homologous interaction sites in the hepatitis B virus core protein. J Virol 72: 4997-5005, 1998.

18. Wynne SA, Crowther RA and Leslie AG: The crystal structure of the human hepatitis B virus capsid. Mol Cell 3: 771-780, 1999.

19. Ponsel D and Bruss V: Mapping of amino acid side chains on the surface of hepatitis B virus capsids required for envelopment and virion formation. J Virol 77: 416-422, 2003.

20. Pairan A and Bruss V: Functional surfaces of the hepatitis B virus capsid. J Virol 83: 11616-11623, 2009.

21. Günther S, Sommer G, Von Breunig F, Iwanska A, Kalinina T, Sterneck $\mathrm{M}$ and Will $\mathrm{H}$ : Amplification of full-length hepatitis B virus genomes from samples from patients with low levels of viremia: Frequency and functional consequences of PCR-introduced mutations. J Clin Microbiol 36: 531-538, 1998.

22. Singh M, Dicaire A, Wakil AE, Luscombe C and Sacks SL: Quantitation of hepatitis B virus (HBV) covalently closed circular DNA (cccDNA) in the liver of HBV-infected patients by LightCycler real-time PCR. J Virol Methods 118: 159-167, 2004.

23. Hayer J, Jadeau F, Deléage G, Kay A, Zoulim F and Combet C: HBVdb: A knowledge database for hepatitis B virus. Nucleic Acids Res 41: D566-D570, 2013.

24. Sievers F, Wilm A, Dineen D, Gibson TJ, Karplus K, Li W, Lopez R, McWilliam H, Remmert M, Söding J, et al: Fast, scalable generation of high-quality protein multiple sequence alignments using clustal omega. Mol Syst Biol 7: 539, 2011.

25. Bock CT, Schwinn S, Locarnini S, Fyfe J, Manns MP, Trautwein C and Zentgraf $\mathrm{H}$ : Structural organization of the hepatitis B virus minichromosome. J Mol Biol 307: 183-196, 2001.

26. Yamamoto M, Hayashi N, Takehara T, Ueda K, Mita E, Tatsumi T, Sasaki Y, Kasahara A and Hori M: Intracellular single-chain antibody against hepatitis B virus core protein inhibits the replication of hepatitis B virus in cultured cells. Hepatology 30: 300-307, 1999

27. Butz K, Denk C, Fitscher B, Crnkovic-Mertens I, Ullmann A, Schröder CH and Hoppe-Seyler F: Peptide aptamers targeting the hepatitis B virus core protein: A new class of molecules with antiviral activity. Oncogene 20: 6579-6586, 2001.

28. Mehta A, Conyers B, Tyrrell DL, Walters KA, Tipples GA, Dwek RA and Block TM: Structure-activity relationship of a new class of anti-hepatitis B virus agents. Antimicrob Agents Chemother 46: 4004-4008, 2002.

29. Zlotnick A, Ceres P, Singh S and Johnson JM: A small molecule inhibits and misdirects assembly of hepatitis B virus capsids. J Virol 76: 4848-4854, 2002.

30. Tan Z, Pionek K, Unchwaniwala N, Maguire ML, Loeb DD and Zlotnick A: The interface between hepatitis B virus capsid proteins affects self-assembly, pregenomic RNA packaging, and reverse transcription. J Virol 89: 3275-3284, 2015.

31. Pollicino T, Belloni L, Raffa G, Pediconi N, Squadrito G, Raimondo $\mathrm{G}$ and Levrero M: Hepatitis B virus replication is regulated by the acetylation status of hepatitis B virus cccDNA-bound H3 and H4 histones. Gastroenterology 130: 823-837, 2006.

This work is licensed under a Creative Commons Attribution-NonCommercial-NoDerivatives 4.0 International (CC BY-NC-ND 4.0) License. 